\title{
Atmosfera controlada para o armazenamento de goiaba cultivar 'Paluma'
}

\author{
Auri Brackmann ${ }^{*}$; Rogério de Oliveira Anese ${ }^{2}$ Vanderlei Both $^{3}$; Fabio Rodrigo Thewes ${ }^{4}$ Diniz Fronza ${ }^{5}$
}

\section{RESUMO}

A goiaba é uma fruta que apresenta alta perecibilidade, sendo o armazenamento em Atmosfera Controlada (AC) uma técnica que pode prolongar a vida pós-colheita, mantendo sua qualidade. O objetivo deste trabalho foi determinar a melhor condição de armazenamento em AC para a conservação da qualidade da goiaba, cultivar 'Paluma'. O experimento foi conduzido no Delineamento em Blocos ao Acaso, com os tratamentos: [1] 20,9 $\mathrm{kPa} \mathrm{O}_{2}+0,03 \mathrm{kPa} \mathrm{CO}_{2}\left(\mathrm{AR}_{-}\right.$ testemunha); [2] 1,0 $\mathrm{kPaO}_{2}+2,0 \mathrm{kPaCO}_{2} ;[3] 2,0 \mathrm{kPa} \mathrm{O}_{2}+2,0 \mathrm{kPa} \mathrm{CO}_{2} ;$ [4] 3,0 $\mathrm{kPa} \mathrm{O}_{2}+2,0 \mathrm{kPa} \mathrm{CO}_{2} ; \mathrm{e} \mathrm{[5]} \mathrm{3,0} \mathrm{kPa} \mathrm{O}+4,0$ $\mathrm{kPa} \mathrm{CO}_{2}$. Todos os frutos foram armazenados à temperatura de $8^{\circ} \mathrm{C}( \pm 0,2)$ sob umidade relativa de $95 \%( \pm 2,0)$. Após 28 dias de armazenamento, os frutos foram avaliados quanto às suas características físico-químicas. A melhor conservação da goiaba cultivar 'Paluma' em AC foi obtida com $\mathrm{O}_{2}$ entre 1 e $2 \mathrm{kPa}$, combinado com $2 \mathrm{kPa}$ de $\mathrm{CO}_{2}$. Estas condições reduziram a degradação da coloração verde da epiderme, a perda de firmeza e de acidez e mantiveram a coloração da polpa com mais eficiência; porém, a alta ocorrência de podridões durante a vida de prateleira é o fator que limita o período de conservação desta goiaba.

Palavras-chave: Psidium guajava L., armazenamento, qualidade, oxigênio, gás carbônico.

\section{ABSTRACT}

\section{Controlled atmosphere for storage of 'Paluma' guava}

Guava is a highly perishable fruit. Controlled atmosphere (CA) storage techniques could extend its shelf life while maintaining the quality of fruit. The aim of this work was to determine the best CA conditions for the storage of 'Paluma' guava. The experiment consisted in a randomized block design with the follow treatments: [1] $20.9 \mathrm{kPa} \mathrm{\textrm {O } _ { 2 } +}$ $0.03 \mathrm{kPa} \mathrm{CO}_{2}\left(\mathrm{CS}\right.$ - control); [2] $1.0 \mathrm{kPa} \mathrm{O}_{2}+2.0 \mathrm{kPaCO}_{2}$; [3] $2.0 \mathrm{kPa} \mathrm{O}_{2}+2.0 \mathrm{kPa} \mathrm{CO}_{2} ;$ [4] $3.0 \mathrm{kPa} \mathrm{O}_{2}+2.0 \mathrm{kPa} \mathrm{CO}_{2}$; and [5] $3.0 \mathrm{kPa} \mathrm{O}_{2}+4.0 \mathrm{kPa} \mathrm{CO}_{2}$. Fruit were stored at $8^{\circ} \mathrm{C}( \pm 0.2)$ at a relative humidity of $95 \%( \pm 2.0)$. After 28 days of storage the fruits were evaluated for their physicochemical characteristics. The best CA storage conditions for 'Paluma' guava is 1 and $2 \mathrm{kPa} \mathrm{O}_{2}$ combined with $2 \mathrm{kPa} \mathrm{CO}_{2}$. These conditions reduced the degradation of the peel green color, loss of firmness and acidity, and keeps the flesh color more effectively, but the high occurrence of decay during self-life is the limiting factor of the storage period.

Key words: Psidium guajava L., storage, quality, oxygen, carbon dioxide.

\footnotetext{
Recebido para publicação em 30/07/2010 e aprovado em 10/02/20112

'Engenheiro-Agrônomo, Doutor. Departamento de Fitotecnia da Universidade Federal de Santa Maria, Avenida Roraima, 1000, Bairro Camobi, 97105-900, Santa Maria, Rio Grande do Sul, Brasil. auribrackmann@ gmail.com "autor para correspondência

${ }^{2}$ Graduandos do Curso de Agronomia. Universidade Federal de Santa Maria, Avenida Roraima, 1000, Bairro Camobi, 97105-900, Santa Maria, Rio Grande do Sul, Brasil. Srogerio_anese@yahoo.com.br; fthewes@yahoo.com.br

${ }^{3}$ Engenheiro-Agrônomo, Pós-graduação em Agronomia. Universidade Federal de Santa Maria, Avenida Roraima, 1000, Bairro Camobi, Santa Maria, Rio Grande do Sul, Brasil. vanderleiboth@yahoo.com.br

${ }^{4}$ Engenheiro-Agrônomo, Doutor. Colégio Politécnico da Universidade Federal de Santa Maria, Avenida Roraima, 1000, Bairro Camobi, 97105-900, Santa Maria, Rio Grande do Sul, Brasil. dinizfronza@yahoo.com.br

${ }^{5}$ Engenheiro-Agrônomo, Doutor. Colégio Politécnico da Universidade Federal de Santa Maria, Avenida Roraima, 1000, Bairro Camobi, 97105-900, Santa Maria, Rio Grande do Sul, Brasil. dinizfronza@yahoo.com.br
} 


\section{INTRODUÇAO}

O Brasil encontra-se entre os principais países produtores de goiaba (Psidium guajava L.), com volume anual em torno de 300 mil toneladas, sendo o cultivar 'Paluma' o mais plantado (Pommer et al., 2006). A goiaba é um fruto que apresenta reduzida vida pós-colheita, por apresentar aumento na produção de etileno, taxa respiratória alta, rápida perda de firmeza e incidência de podridões (Jacomino et al., 2003; Singh \& Pal, 2008). Estas características limitam o potencial de armazenamento, comercialização e exportação da goiaba, sendo os fatores responsáveis pelo inexpressivo volume exportado pelo país, que, em 2010 foi de apenas $147.348 \mathrm{~kg}$ (IBRAF, 2011).

A investigação de técnicas que visem à preservação da qualidade da goiaba é de relevada importância, pois, com elas, possibilitar-se-ia maior período de comercialização e, principalmente, permitir-se-ia o transporte dos frutos a longas distâncias, como o transporte marítimo, visando à exportação. Uma técnica que pode ser empregada para estender a vida pós-colheita da goiaba é a da Atmosfera Controlada (AC) (Singh \& Pal, 2008). A AC, uma técnica complementar ao Armazenamento Refrigerado (AR), caracteriza-se pela redução da pressão parcial de oxigênio $\left(\mathrm{O}_{2}\right)$ e elevação da de gás carbônico $\left(\mathrm{CO}_{2}\right)$, bem como pelo controle da concentração desses gases durante a armazenagem (Gorny \& Kader, 1996). O baixo nível de $\mathrm{O}_{2}$ e o alto de $\mathrm{CO}_{2}$ reduzem a produção de etileno (Gorny \& Kader, 1996) e a respiração, bem como os demais processos dependentes destes, como perda de firmeza, redução da coloração verde da epiderme e diminuição da acidez.

Os benefícios e as melhores condições de AC para manutenção da qualidade pós-colheita de frutas foram comprovados, para várias espécies e cultivares, como maçãs (Brackmann et al., 2008), peras (Lara et al., 2003), pêssegos (Nava \& Brackmann, 2002) e alguns cultivares de goiaba (Singh \& Pal, 2008). Jacomino et al. (2001) constataram que a qualidade de goiabas cultivar 'Kumagai', armazenadas em Atmosfera Modificada (AM), manteve-se melhor na condição em que a concentração interna de gases estabilizou-se em $0,5 \mathrm{kPa}$ de $\mathrm{O}_{2}$ e 4,5 $\mathrm{kPa}$ de $\mathrm{CO}_{2}$. No que diz respeito ao armazenamento em AC de goiaba cultivar 'Paluma', não existem informações na literatura. Kader (2009) recomenda que o nível de $\mathrm{O}_{2}$ ideal para goiabas está entre 2 e 5 kPa. Para os cultivares 'Lucknow-49', 'Allahabad Safeda' e 'Apple Colour', Singh \& Pal (2008) observaram que as pressões parciais que mantiveram mais satisfatoriamente a qualidade dos frutos foram $5 \mathrm{kPaO}_{2}+2,5 \mathrm{kPaCO}_{2}, 5 \mathrm{kPaO}_{2}+5$ $\mathrm{kPaCO}_{2}$ e $8 \mathrm{kPaO}_{2}+5 \mathrm{kPaCO}_{2}$, respectivamente. Por causa da diferença de comportamento entre as variedades, estes autores recomendam que as pressões parciais de gases sejam determinadas para cada cultivar. Portanto, a determinação das melhores pressões parciais de gases para o armazenamento em AC de goiaba cultivar 'Paluma', visan- do à manutenção da qualidade desta fruta, durante o transporte marítimo e comercialização em países importadores, é de extrema importância para o Brasil.

Diante do exposto, o objetivo deste trabalho foi determinar a melhor condição de armazenamento em Atmosfera Controlada (AC) para a conservação da qualidade da goiaba cultivar 'Paluma'.

\section{MATERIAL E MÉTODOS}

O experimento foi conduzido no Núcleo de Pesquisa em Pós-colheita (NPP) do Departamento de Fitotecnia da Universidade Federal de Santa Maria (UFSM), com goiabas do cultivar 'Paluma' provenientes de pomar localizado no Colégio Politécnico da UFSM, Santa Maria (RS). Após a colheita, os frutos foram transportados até o NPP, onde foram selecionados, eliminando-se aqueles com lesões, defeituosos e muito grandes ou muito pequenos. Em seguida, as amostras experimentais foram homogeneizadas. Antes da instalação dos experimentos, os frutos apresentavam as seguintes características de maturação: firmeza da polpa: 90,1 N, ácido ascórbico: 56,2 mg 100g-1, acidez: 11,5 meq $100 \mathrm{~mL}^{-1}$, sólidos solúveis: $7,81^{\circ}$ Brix, cor da polpa: $\mathrm{L}$ de 61,2 e hue de $35,2^{\circ}$, cor da epiderme: hue de $109,9^{\circ}$, produção de etileno: $0,5885 \mu \mathrm{L} \mathrm{C}_{2} \mathrm{H}_{4} \mathrm{~kg}^{-1} \mathrm{~h}^{-1}$, respiração: $19,06 \mathrm{~mL} \mathrm{CO}_{2} \mathrm{~kg}^{-1} \mathrm{~h}^{-1}$. O experimento foi conduzido no Delineamento em Blocos ao Acaso (DBA), com cinco tratamentos e cinco repetições, sendo cada unidade experimental composta de doze frutos.

As condições de Atmosfera Controlada avaliadas foram: [1] 20,9 $\mathrm{kPa} \mathrm{O}_{2}+0,03 \mathrm{kPa} \mathrm{CO}_{2}(\mathrm{AR}$ - testemunha); [2] $1,0 \mathrm{kPaO}_{2}+2,0 \mathrm{kPaCO}_{2}$; [3] 2,0 $\mathrm{kPaO}_{2}+2,0 \mathrm{kPaCO}_{2}$; [4] $3,0 \mathrm{kPaO}_{2}+2,0 \mathrm{kPaCO}_{2}$; e [5] 3,0 $\mathrm{kPaO}_{2}+4,0 \mathrm{kPaCO}_{2}$. Todos os frutos foram armazenados à temperatura de $8^{\circ} \mathrm{C}$ $( \pm 0,2)$, sob umidade relativa de $95 \%( \pm 2,0)$. Os frutos foram armazenados em minicâmaras experimentais de AC, com volume de $0,08 \mathrm{~m}^{3}$, que permaneceram no interior de uma câmara fria de $45 \mathrm{~m}^{3}$. As condições de atmosfera controlada foram obtidas mediante a redução dos níveis de $\mathrm{O}_{2}$ com a injeção de nitrogênio nas minicâmaras e, a concentração de $\mathrm{CO}_{2}$, por meio da injeção, nelas, deste gás, até ao nível pré-estabelecido em cada tratamento. A manutenção das concentrações dos gases foi realizada por meio da análise diária por analisador eletrônico de gases, marca Kronenberger. $\mathrm{O} \mathrm{O}_{2}$ consumido pela respiração foi reposto pela adição de ar atmosférico e o excesso de $\mathrm{CO}_{2}$ absorvido com solução de hidróxido de potássio.

Depois de transcorridos 28 dias de armazenamento, os frutos foram retirados das minicâmaras e expostos à temperatura de $20^{\circ} \mathrm{C}$, durante 3 dias, simulando o período de comercialização. As variáveis analisadas foram:

a) firmeza de polpa: com auxílio de um penetrômetro com ponteira de 7,9 mm de diâmetro, sendo os resultados expressos em Newtons $(\mathrm{N})$; 
b) ácido ascórbico: determinado pela titulação, com iodato de potássio $(\mathrm{K}) 0,01 \mathrm{~N}$, de uma solução contendo $12 \mathrm{~g}$ de suco de goiaba, $20 \mathrm{~mL}$ de ácido sulfúrico a 20\%, 1 $\mathrm{mL}$ de iodeto de potássio a $10 \%$ e $1 \mathrm{~mL}$ de solução de amido a $1 \%$. Para o cálculo, utilizou-se a seguinte fórmula: ácido ascórbico $=(100 \times \mathrm{V} \times \mathrm{F}) / \mathrm{P}$, em que V = volume gasto da solução de iodato de $\mathrm{K}(\mathrm{mL}) ; \mathrm{F}$ = fator de equivalência da solução de iodato de $\mathrm{K}$ em função da normalidade $(0,8806)$ e $\mathrm{P}=$ massa da amostra $(\mathrm{g})$. Os resultados foram expressos em mg de ácido ascórbico por $100 \mathrm{~g}$ de polpa;

c) acidez total titulável: pela titulação de $10,0 \mathrm{~mL}$ de suco com $\mathrm{NaOH} 0,1 \mathrm{~N}$ até $\mathrm{pH} 8,1$, sendo os resultados apresentados em meq100 $\mathrm{mL}^{-1}$;

d) sólidos solúveis (SS): por refratometria, com resultados expressos em graus Brix ( ${ }^{\circ}$ Brix);

e) cor da epiderme e da polpa: determinadas com um colorímetro marca Minolta, modelo CR 310, que utiliza um sistema tridimensional de cores. Os resultados foram expressos pelo eixo vertical "L", o qual vai de zero a 100 , sendo o valor zero totalmente preto e 100 totalmente branco, e ângulo hue, o qual mostra a localização da cor em um diagrama, em que o ângulo $0^{\circ}$ representa vermelho puro; $90^{\circ}$ representa o amarelo puro; $180^{\circ}$, o verde puro e $270^{\circ}$, o azul;

f) incidência de podridões: por observação e contagem dos frutos que apresentavam lesões maiores do que $0,5 \mathrm{~cm}$, causadas por podridões, resultados expressos em percentagem;

g) perda de massa: obtida por cálculo da diferença de peso dos frutos antes e depois do armazenamento, expressa em percentagem de perda de massa;

h) atividade da ACC (ácido 1-carboxílico-1-aminociclopropano) oxidase: por meio da retirada de amostras de três gramas de casca da região equatorial dos frutos de cada unidade experimental. Estas foram imediatamente para uma solução contendo $0,1 \mathrm{mM}$ de ACC em $10 \mathrm{mM}$ do tampão MES (ácido 2 (N-morfolino) etanossulfônico), em pH 6,0. Após 30 minutos, as amostras foram acondicionadas em seringas herméticas de $50 \mathrm{~mL}$, nas quais adicionou-se $1 \mathrm{~mL}$ de $\mathrm{CO}_{2}$. Decorridos 30 minutos, a concentração de etileno presente nas seringas foi determinada, sendo os dados expressos em $\mu \mathrm{L} \mathrm{C}_{2} \mathrm{H}_{4} \mathrm{~kg}^{-1} \mathrm{~h}^{-1}$, conforme metodologia de Bufler (1986);

i) produção de etileno: determinada pela injeção de duas amostras de gás de $1 \mathrm{~mL}$, provenientes de cada recipiente de $5000 \mathrm{~mL}$, em um cromatógrafo a gás, marca Varian, modelo 3400, equipado com um detector de ionização por chama (FID) e coluna Porapak N80/100. As temperaturas da coluna, do injetor e do detector foram de 90, 140 e $200^{\circ} \mathrm{C}$, respectivamente. Foi calculada a síntese de etileno em $\mu \mathrm{L} \mathrm{C}_{2} \mathrm{H}_{4} \mathrm{~kg}^{-1} \mathrm{~h}^{-1}$ por meio da concentração de etileno, da massa do fruto, do volume do espaço livre no recipiente e do tempo de fechamento;

j) respiração: determinada pela produção de $\mathrm{CO}_{2}$, com um analisador marca Kronenberger, dos frutos do mesmo frasco utilizado para determinação da produção de etileno, os resultados foram expressos em mL CO $\mathrm{kg}^{-1} \mathrm{~h}^{-1}$.

Para cada variável estudada, foi efetuada análise de variância, sendo as médias comparadas pelo teste de Tukey, com 5\% de probabilidade de erro, exceto para as variáveis produção de etileno, respiração e cor da epiderme, as quais foram analisadas pela Diferença Mínima Significativa $\left(\mathrm{DMS}_{0,05 \%}\right)$. A variável podridão, expressa em percentagem, foi transformada pela fórmula $\operatorname{arc} . \operatorname{sen} \sqrt{(\mathrm{x} / 100)}$, antes da análise de variância.

\section{RESULTADOS E DISCUSSÕES}

Houve um declínio acentuado na firmeza da polpa dos frutos da testemunha, comparada à dos armazenados em Atmosfera Controlada (AC), sendo que a AC com pressão parcial de $\mathrm{O}_{2}$ mais baixo $\left(1,0 \mathrm{kPa}\right.$ de $\mathrm{O}_{2}+2,0 \mathrm{kPa}$ de $\mathrm{CO}_{2}$ ) proporcionou menor perda de firmeza (Tabela 1). A baixa concentração de $\mathrm{O}_{2}$ exerceu grande influência na retenção da firmeza, pois, à medida que foi utilizado um nível maior de $\mathrm{O}_{2}$, houve maior perda. Por outro lado, o nível de $\mathrm{CO}_{2}$ não influenciou esta variável. Trabalhando com outros cultivares, Singh \& Pal (2008) também constataram que a $\mathrm{AC}$ foi eficiente para reduzir a perda de firmeza, e que o $\mathrm{CO}_{2}$ não influenciou esta característica. Provavelmente, a baixa produção de etileno pelos frutos mantidos com menor nível de $\mathrm{O}_{2}$ (Figura 1) tenha sido crucial na redução da ativação de enzimas que degradam os componentes da parede celular, como a poligalacturonase, pectinametilesterase, entre outras (Payasi et al., 2009), as quais estão associadas com a perda de firmeza de frutos.

O teor de ácido ascórbico apresentou considerável aumento durante o período de armazenamento, em todos os tratamentos avaliados (Tabela 1). Entre as condições de AC testadas, não houve diferença significativa, porém, houve uma tendência de os tratamentos com pressão parcial de $\mathrm{O}_{2}$ mais reduzido apresentarem maior aumento no teor de ácido ascórbico. Este aumento parece estar relacionado com a necessidade de remoção de espécies reativas de oxigênio (EROs) que são produzidas durante o amadurecimento (Mondal et al., 2009). Outros autores também encontraram aumento no teor de ácido ascórbico durante o armazenamento de goiabas (Pereira et al., 2005; Cerqueira et al., 2009).

A acidez titulável manteve-se mais alta no tratamento com $1,0 \mathrm{kPa}$ de $\mathrm{O}_{2}+2,0 \mathrm{kPa}$ de $\mathrm{CO}_{2}$. Nos demais tratamentos, houve maior redução da acidez (Tabela 1). Provavelmente, a manutenção da acidez com nível mais baixo de $\mathrm{O}_{2}$ está relacionada com a menor respiração apresentada pelos frutos deste tratamento (Figura 1), pois a perda de 
acidez está diretamente ligada ao processo natural de maturação, em que os ácidos orgânicos são usados como substratos no ciclo dos ácidos tricarboxílicos da atividade respiratória. Houve pequena variação no teor de sólidos solúveis entre tratamentos, mantendo-se mais alta no tratamento com $3,0 \mathrm{kPa}$ de $\mathrm{O}_{2}+2,0 \mathrm{kPa}$ de $\mathrm{CO}_{2}$. Resultados semelhantes foram encontrados por Singh \& Pal (2008). Durante o processo normal de maturação ocorre declínio no teor de ácidos e aumento no teor de açúcares (Bashir \& Abu-goukh, 2003).
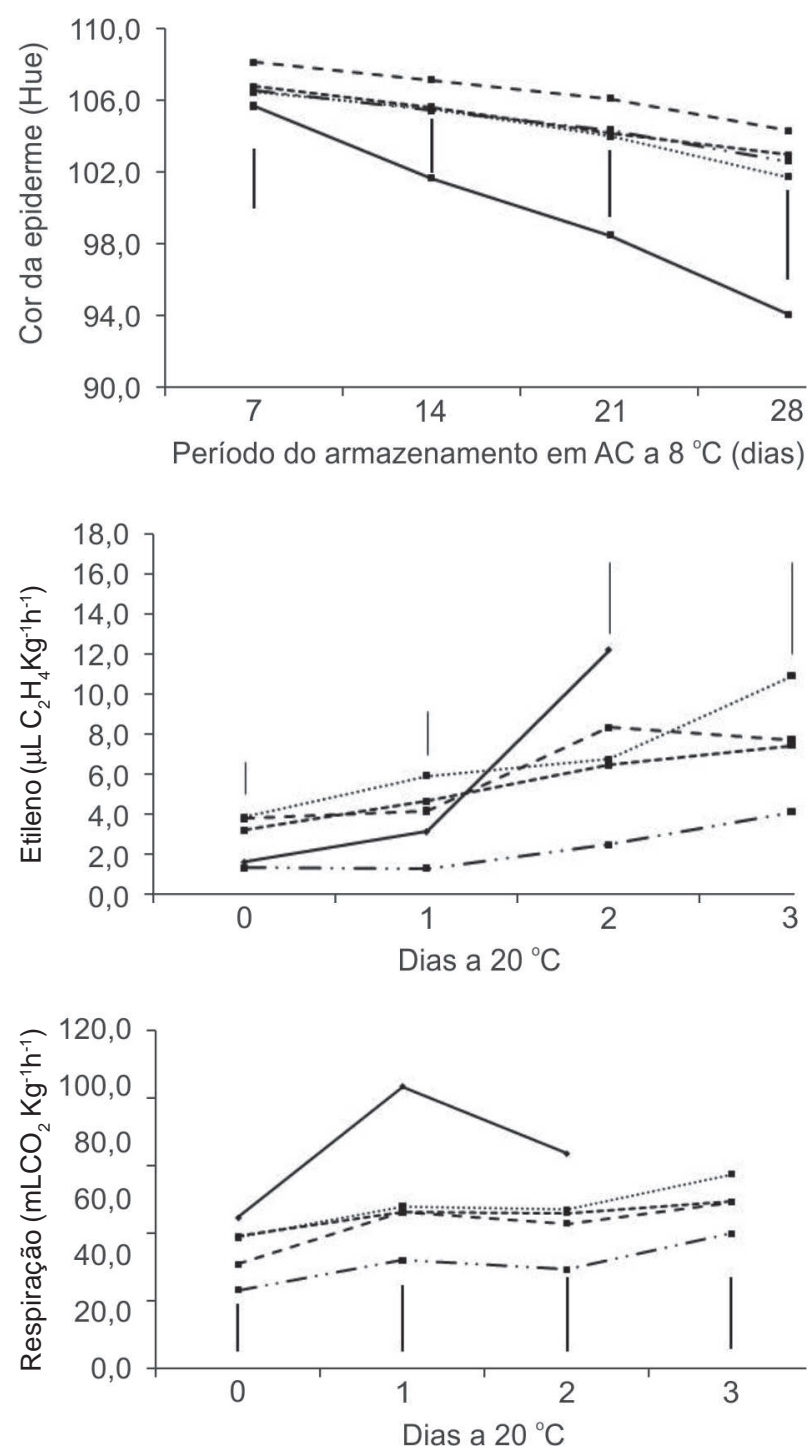

$-20,9 \mathrm{kPa} O 2+0,03 \mathrm{kPa}$ de $\mathrm{CO} 2-\cdots-1 \mathrm{kPa} \mathrm{O} 2+2 \mathrm{kPa} \mathrm{CO} 2$ $-\cdots-2 \mathrm{kPa} \mathrm{O} 2+2 \mathrm{kPa} \mathrm{CO} 2 \quad \cdots---3 \mathrm{kPa} \mathrm{O} 2+2 \mathrm{kPa} \mathrm{CO} 2$ (........... $3 \mathrm{kPa} \mathrm{O} 2+4 \mathrm{kPa} \mathrm{CO} 2$

Figura 1. Cor da epiderme $\left({ }^{\circ} h u e\right)$, produção de etileno $(\mu \mathrm{L}$ $\left.\mathrm{C}_{2} \mathrm{H}_{4} \mathrm{~kg}^{-1} \mathrm{~h}^{-1}\right)$ e respiração $\left(\mathrm{mL} \mathrm{CO} \mathrm{kg}^{-1} \mathrm{~h}^{-1}\right)$ de goiaba 'Paluma' submetida à atmosfera controlada durante 28 dias a $8^{\circ} \mathrm{C}$ e mais 3 dias de exposição à temperatura ambiente $\left(20^{\circ} \mathrm{C}\right)$. Barras verticais indicam Diferença Mínima Significativa $\left(\mathrm{DMS}_{0.05 \%}\right)$ entre tratamentos.
A cor da polpa apresentou-se menos escurecida no tratamento com $\mathrm{O}_{2}$ mais baixo $\left(1,0 \mathrm{kPa}\right.$ de $\mathrm{O}_{2}+2,0 \mathrm{kPa}$ de $\mathrm{CO}_{2}$ ), o que é evidenciado pelo índice L (Tabela 2). A medida que a pressão parcial de $\mathrm{O}_{2}$ aumentou, houve maior escurecimento da polpa. Segundo Kader (2009), o escurecimento da polpa é considerado um dos sintomas de dano por frio. Portanto, a AC foi eficiente na redução da incidência deste distúrbio em goiabas. A AC com nível de $\mathrm{O}_{2}$ mais baixo reduziu a intensificação da cor vermelha da polpa durante o armazenamento, o que é evidenciado pelo maior valor do ângulo hue. Entretanto, não houve diferença significativa $(p>0,05)$ entre a testemunha e os demais tratamentos, o que, provavelmente, esteja relacionado com a interferência do alto escurecimento apresentado pela testemunha, na determinação do ângulo hue.

Na avaliação realizada quando os frutos saíram da câmara, a incidência de podridões foi menor em todos os tratamentos em AC (Tabela 2). Já aos três dias de exposição à temperatura de $20^{\circ} \mathrm{C}$, todos os tratamentos apresentaram elevado percentual de frutos com lesões maiores do que $0,5 \mathrm{~cm}$, causadas por podridões, sendo que os frutos da testemunha apresentaram-se totalmente podres. Nas condições de AC, o menor metabolismo dos frutos, provavelmente, possibilitou maior integridade dos tecidos, conferindo maior resistência das células à penetração de agentes causadores de podridões. A alta incidência de podridões aos 3 dias, a $20^{\circ} \mathrm{C}$, evidencia que o consumo dos frutos deve ser realizado em um período de

Tabela 1. Firmeza da polpa, ácido ascórbico, acidez e sólidos solúveis de goiaba cultivar 'Paluma' submetida à atmosfera controlada durante 28 dias a $8^{\circ} \mathrm{C}$ mais 3 dias de exposição à temperatura ambiente $\left(20^{\circ} \mathrm{C}\right)$

\begin{tabular}{|c|c|c|}
\hline $\begin{array}{l}\text { Tratamentos } \\
\mathrm{O}_{2}+\mathrm{CO}_{2}(\mathrm{kPa})\end{array}$ & $\begin{array}{c}\text { Firmeza da polpa } \\
(\mathbf{N})\end{array}$ & $\begin{array}{l}\text { Ácido Ascórbico } \\
\left(\mathrm{mg} \mathrm{100g}^{-1}\right)\end{array}$ \\
\hline Análise inicial & 90,1 & 56,2 \\
\hline $20,9+0,03$ & $12,7 \quad c^{*}$ & $66,7 \mathrm{~b}$ \\
\hline $1,0+2,0$ & $59,0 \mathrm{a}$ & $93,5 \mathrm{a}$ \\
\hline $2,0+2,0$ & $38,3 \mathrm{~b}$ & $87,8 \mathrm{a}$ \\
\hline $3,0+2,0$ & $31,5 \mathrm{~b}$ & $77,3 \mathrm{ab}$ \\
\hline $3,0+4,0$ & $31,9 \mathrm{~b}$ & $76,9 a b$ \\
\hline \multirow[t]{2}{*}{$\mathrm{CV}(\%)$} & 15,6 & 11,3 \\
\hline & $\begin{array}{c}\text { Acidez } \\
\left(\text { meq } 100 \mathrm{~mL}^{-1}\right)\end{array}$ & $\begin{array}{c}\text { S S } \\
\left({ }^{\circ} \text { Brix }\right)\end{array}$ \\
\hline Análise inicial & 11,5 & 7,82 \\
\hline $20,9+0,03$ & $7,76 \mathrm{~b}$ & $7,50 \mathrm{~b}$ \\
\hline $1,0+2,0$ & $10,7 \mathrm{a}$ & $7,26 \mathrm{~b}$ \\
\hline $2,0+2,0$ & $8,18 \mathrm{~b}$ & $7,78 \mathrm{ab}$ \\
\hline $3,0+2,0$ & $7,82 \mathrm{~b}$ & $8,12 \mathrm{a}$ \\
\hline $3,0+4,0$ & $7,25 \mathrm{~b}$ & $7,80 \mathrm{ab}$ \\
\hline $\mathrm{CV}(\%)$ & 9,11 & 3,77 \\
\hline
\end{tabular}

"Médias seguidas pela mesma letra não apresentam diferença estatística significativa pelo teste Tukey a $5 \%$ de probabilidade de erro. 
Tabela 2. Cor da polpa, podridões, perda de massa e ACC oxidase de goiaba cultivar 'Paluma' submetida à atmosfera controlada durante 28 dias a $8^{\circ} \mathrm{C}$ mais 3 dias de exposição à temperatura ambiente $\left(20^{\circ} \mathrm{C}\right)$

\begin{tabular}{|c|c|c|c|c|}
\hline \multirow{2}{*}{$\begin{array}{c}\text { Tratamentos } \\
\mathrm{O}_{2}+\mathrm{CO}_{2}(\mathrm{kPa})\end{array}$} & \multicolumn{2}{|c|}{ Cor da polpa } & \multicolumn{2}{|c|}{ Podridões (\%) } \\
\hline & (L) & $\left({ }^{\circ}\right.$ hue $)$ & Saída & 3 dias a $20^{\circ} \mathrm{C}$ \\
\hline Análise inicial & 61,2 & 35,2 & - & - \\
\hline $20,9+0,03$ & $49,9 \quad \mathrm{~d}^{*}$ & $33,6 \mathrm{ab}$ & $79,9 \mathrm{a}$ & 100,0 a \\
\hline $1,0+2,0$ & $58,3 \mathrm{a}$ & $34,6 \mathrm{a}$ & $15,0 \mathrm{~b}$ & $79,8 \mathrm{ab}$ \\
\hline $2,0+2,0$ & $55,6 \mathrm{~b}$ & $32,2 \mathrm{bc}$ & $28,5 \mathrm{~b}$ & $72,0 \mathrm{~b}$ \\
\hline $3,0+2,0$ & $52,7 \mathrm{c}$ & $31,5 \quad \mathrm{c}$ & $30,7 \mathrm{~b}$ & $76,8 \mathrm{~b}$ \\
\hline $3,0+4,0$ & $51,6 \quad \mathrm{~d}$ & $30,1 \quad \mathrm{c}$ & $44,2 \mathrm{~b}$ & $84,9 \mathrm{ab}$ \\
\hline \multirow[t]{2}{*}{$\mathrm{CV}(\%)$} & 2,12 & 3,1 & 20,3 & 12,9 \\
\hline & \multicolumn{2}{|c|}{ Perda de massa $(\%)$} & \multicolumn{2}{|c|}{ ACC oxidase $\left(\mu \mathrm{L} \mathrm{C}_{2} \mathrm{H}_{4} \mathrm{~kg}^{-1} \mathrm{~h}^{-1}\right)$} \\
\hline Análise inicial & \multicolumn{2}{|c|}{-} & \multicolumn{2}{|c|}{0,017} \\
\hline $20,9+0,03$ & \multicolumn{2}{|c|}{$1,8 \mathrm{a}$} & \multicolumn{2}{|c|}{$0,011 \mathrm{a}$} \\
\hline $1,0+2,0$ & \multicolumn{2}{|c|}{$1,4 \mathrm{a}$} & \multicolumn{2}{|c|}{0,026 a } \\
\hline $2,0+2,0$ & \multicolumn{2}{|c|}{$1,6 \mathrm{a}$} & \multicolumn{2}{|c|}{$0,032 \mathrm{a}$} \\
\hline $3,0+2,0$ & \multicolumn{2}{|c|}{$1,7 \mathrm{a}$} & \multicolumn{2}{|c|}{0,031 a } \\
\hline $3,0+4,0$ & \multicolumn{2}{|c|}{$1,6 \mathrm{a}$} & \multicolumn{2}{|c|}{$0,033 \mathrm{a}$} \\
\hline $\mathrm{CV}(\%)$ & \multicolumn{2}{|c|}{11,7} & \multicolumn{2}{|c|}{36,8} \\
\hline
\end{tabular}

"Médias seguidas pela mesma letra não apresentam diferença estatística significativa pelo teste Tukey a 5\% de probabilidade de erro.

tempo inferior, quando mantidos na temperatura de $20^{\circ} \mathrm{C}$, após serem retirados do armazenamento em AC. Resultados semelhantes foram reportados por Singh \& Pal (2008) na ocorrência de podridões. Há necessidade de futuros trabalhos a fim de estudar o efeito da redução da temperatura e do controle da podridão, durante o armazenamento em AC de goiabas por longos períodos.

As variáveis perda de massa e atividade da ACC oxidase não apresentaram diferença estatística entre os tratamentos (Tabela 2).

Com relação à cor da epiderme, houve maior redução da cor verde durante o período de armazenamento nos frutos da testemunha (AR), comparada à dos de AC, de acordo com o valor do ângulo hue (Figura 1). A condição de $\mathrm{AC}$ de 2,0 $\mathrm{kPa} \mathrm{O}_{2}+2,0 \mathrm{kPa}$ de $\mathrm{CO}_{2}$ foi a que manteve a cor verde da epiderme com mais eficiência, entretanto não diferiu estatisticamente $(p>0,05)$ das demais condições de AC. Para Beaudry (1999), o efeito do baixo nível de $\mathrm{O}_{2}$ e elevado nível de $\mathrm{CO}_{2}$ está na sensibilidade dos tecidos ao etileno, o qual tem ação na ativação de enzimas, como a clorofilase, que degradam a clorofila. Trabalhando com pêssegos cultivar 'Chiripá', Nava \& Brackmann (2002) observaram que a AC reduziu a degradação da clorofila da epiderme, mantendo a coloração verde.

A produção de etileno foi menor no tratamento com nível de $\mathrm{O}_{2}$ mais baixo $(1 \mathrm{kPa})$, durante o período de exposição a $20^{\circ} \mathrm{C}$ (Figura 1). O nível de $\mathrm{CO}_{2}$ não exerceu influência na produção de etileno nem na respiração dos frutos. A AC reduziu a produção de etileno de três cultivares de goiaba, sendo que o baixo nível de $\mathrm{O}_{2}$ foi mais efetivo, nessa redução, do que o alto nível de $\mathrm{CO}_{2}$ (Singh \& Pal, 2008). Provavelmente, os níveis de gases tenham reduzi- do a atividade de enzimas da rota de síntese do etileno (Gorny \& Kader, 1996). Da mesma forma que a produção de etileno, a respiração das goiabas foi menor quando os frutos estavam submetidos a $1,0 \mathrm{kPa}$ de $\mathrm{O}_{2}$ (Figura 1). Talvez o alto nível de $\mathrm{CO}_{2}$ não tenha exercido grande influência na respiração e na produção de etileno, pelo fato de este estar associado a uma condição de $\mathrm{O}_{2}$ mais elevada $(3 \mathrm{kPa})$.

\section{CONCLUSÃO}

As melhores pressões parciais de gases para o armazenamento em AC de goiaba cultivar 'Paluma' estão entre 1 e $2 \mathrm{kPa}$ de $\mathrm{O}_{2}$, combinado com $2 \mathrm{kPa}$ de $\mathrm{CO}_{2}$. Estas condições reduzem a degradação da coloração verde da epiderme, a perda de firmeza e de acidez, e mantém a coloração da polpa com mais eficiência; porém não controlam a ocorrência de podridões durante 3 dias a $20^{\circ} \mathrm{C}$, durante a vida de prateleira. Novos trabalhos, avaliando temperaturas de armazenamento mais baixas e controle da podridão, durante o armazenamento em AC, são necessários.

\section{REFERÊNCIAS}

Bashir HA \& Abu-goukh AA (2003) Compositional changes during guava fruit ripening. Food Chemistry, 80:557-563.

Beaudry RM (1999) Effect of $\mathrm{O}_{2}$ and $\mathrm{CO}_{2}$ partial pressure on selected phenomena affecting fruit and vegetable quality. Postharvest Biology and Technology, 15:293-303.

Brackmann A, Weber A, Pinto JAV, Neuwald AD \& Steffens CA (2008) Manutenção da qualidade pós-colheita de maçãs 'Royal Gala' e 'Galaxy' sob armazenamento em atmosfera controlada. Ciência rural, 38:2478-2484.

Rev. Ceres, Viçosa, v. 59, n.2, p. 151-156, mar/abr, 2012 
Bufler G (1986) Ethylene-promoted conversion of 1aminocyclopropane-1-carboxylic acid to ethylene in peel of apple at various stages of fruit development. Plant Physiology, 80:539-543.

Cerqueira TS, Jacomino AP, Sasaki FF \& Amorin L (2009) Controle do amadurecimento de goiabas 'Kumagai' tratadas com 1metilciclopropeno. Revista brasileira de fruticultura, 31:687692.

Gorny JR \& Kader AA (1996) Controlled atmosphere suppression of ACC synthase and ACC oxidase in 'Golden Delicious' apples during long-term cold storage. Journal American Society for Horticultural Science, 121:751-755.

Instituto Brasileiro de Frutas - IBRAF (2011) Comparativo das exportações brasileiras de frutas frescas 2009-2010. Disponível em: <http://www.ibraf.org.br/estatisticasExporta\%C3\% A 7\% $3 \%$ A 3o/Comparativo_das_Exporta\%C3\%A7\% C3\% B5es_Brasileiras_de_Frutas_frescas_2010-2009.pdf $>$ Acessado em: 29 de setembro de 2011 .

Jacomino AP, Ojeda RM, Kluge RA \& Scarpare Filho JA (2003) Conservação de goiabas tratadas com emulsões de cera de carnaúba. Revista brasileira de fruticultura, 25:401-405.

Jacomino AP, Sarantópoulos CIGL, Sigrist JMM, Kugle RA \& Minami K (2001) Sensorial characteristics of "Kumagai" guavas submitted to passive modified atmosphere in plastic packages. Journal of plastic film and sheeting, 17:6-21.

Kader AA (2009) Guava: recommendations for maintaining postharvest quality. Produces facts, California. Disponível em: http://postharvest.ucdavis.edu/Produce/ProduceFacts/Fruit/ Guava.shtml. Acessado em: 01 de abril de 2010.
Lara I, Miró RM, Fuentes T, Sayez G, Graell J \& López ML (2003) Biosynthesis of volatile aroma compounds in pear fruit stored under long-term controlled-atmosphere conditions. Postharvest Biology and technology, 29:29-39.

Mondal K, Malhotra SP, Jain V \& Singh R (2009) Oxidative stress and antioxidant systems in Guava (Psidium guajava L.) fruits during ripening Physiology and Molecular Biology of Plants, $15: 327-334$

Nava GA \& Brackmann A (2002) Armazenamento de pêssegos (Prunus persica (L.) Batsch), cv. Chiripá, em atmosfera controlada. Revista brasileira de fruticultura, 24:328-332.

Payasi A, Mishra NN, Chaves ALS \& Singh R (2009) Biochemistry of fruit softening: an overview. Physiology and Molecular Biology of Plants, 15:103-113.

Pereira T, Carlos LA, Oliveira JG \& Monteiro AR (2005) Características físicas e químicas de goiabas cv. Cortibel (Psiduim guajava) estocadas sob refrigeração em filmes X-Tend. Alimentos e Nutricão, 16:11-16.

Pommer CV, Murakami KNR \& Watlington F (2009) Goiaba no mundo. O Agronômico, 58:22-26.

Singh SP \& Pal RK (2008) Controlled atmosphere storage of guava (Psidium guajava L.) fruit. Postharvest Biology and technology, 47:296-306. 